\title{
Controlling light with light in silver-nanospheres and gold-nanorods colloids ${ }^{\diamond}$
}

\section{Controlando luz con luz en coloides de nanoesferas de plata y nanobastones de oro}

\author{
Cid B. de Araújo,*, Albert S. Reyna** and Nathália Talita C. Oliveira***
}

\begin{abstract}
A review is presented on recent research that demonstrate the control of light-bylight in colloids containing silver-nanospheres (Ag-NS) and gold-nanorods (Au-NR). The presentation is based on experiments performed with pulsed lasers by exploiting the ultrafast electronic nonlinearity of samples exhibiting cubic-quintic nonlinearities. Guiding and confinement of light induced by optical vortex solitons in colloidal suspensions of Ag-NS, and nonlinear processes of light scattering, absorption and refraction in colloids with Au-NR were investigated in the experiments. The results are analyzed by numerical simulations based on modified nonlinear Schrödinger equations. The developments herein discussed are in the forefront of interest for plasmonic applications with metal nanoparticles.
\end{abstract}

KEYWORDS: metal nanoparticles, plasmonics, nanospheres, nanorods, vortex solitons, nonlinear absorption, nonlinear scattering, nonlinear refraction.

RESUMEN: Este artículo presenta una revisión sobre investigaciones recientes que demuestran el control de la luz por luz en coloides que contienen nanoesferas de plata (Ag-NS) y nanobastones de oro (Au-NR). La presentación se basa en experimentos realizados con láseres pulsados mediante la explotación de la no linealidad electrónica ultrarrápida de las muestras que exhiben no linealidades cúbico-quínticas. Efectos tales como el guiamiento y confinamiento de la luz inducida por solitones ópticos, con estructura vortical, en suspensiones coloidales de AgNS, además de procesos no lineales de dispersión, absorción y refracción de la luz en coloides con Au-NR fueron investigados en los experimentos. Los resultados fueron analizados mediante simulaciones numéricas basadas en adaptaciones de la ecuación de Schrödinger no lineal. Los avances discutidos en el presente artículo están a la vanguardia del interés en aplicaciones plasmónicas con nanopartículas metálicas.

Recibido: 15 de agosto de 2019.

Aceptado: 28 de septiembre de 2019.

${ }^{\bullet}$ Acknowledgements: We acknowledge financial support by the Coordenação de Aperfeiçoamento de Pessoal de Nível Superior (CAPES), Conselho Nacional de Desenvolvimento Científico e Tecnológico (CNPq), and Fundação de Amparo à Ciência e Tecnologia do Estado de Pernambuco (FACEPE). The work was performed in the framework of the National Institute of Photonics (INCT de Fotônica).

*Departamento de Física, Universidade Federal de Pernambuco, 50670-901 Recife, PE, Brazil. Programa de Pós-Graduação em Ciência de Materiais, Universidade Federal de Pernambuco, 50670-901 Recife, PE, Brazil.

**Programa de Pós-Graduação em Engenharia Física, Unidade Acadêmica do Cabo de Santo Agostinho, Universidade Federal Rural de Pernambuco, 54518-430 Cabo de Santo Agostinho, PE, Brazil.

****Programa de Pós-Graduação em Ciência de Materiais, Universidade Federal de Pernambuco, 50670-901 Recife, PE, Brazil.

'Corresponding author: cid@df.ufpe.br 
PALABRAS CLAVE: nanopartículas metálicas, plasmónica, nanoesferas, nanobastones, solitones vorticales, absorción no lineal, dispersión no lineal, refracción no lineal.

\section{Introduction}

The interest in metal-dielectric composites (MDC), containing gold (Au), silver $(\mathrm{Ag})$ and copper $(\mathrm{Cu})$ nanoparticles (NPs) dates back to ancient times (see, for example, Savage, 1975). One famous example of utensils made of MDC is the Lycurgus cup, manufactured in the 4th century AD, which looks green in the reflected light but appears red in the transmitted light. The colors are due to $\mathrm{Au}-\mathrm{Ag} \mathrm{NPs}$ embedded in the glass. Other well-known example illustrates the interest of artists in decoration of glass objects since long time. Many Middle Age cathedrals exhibit colored stained glass due to $\mathrm{Au}, \mathrm{Ag}$ and $\mathrm{Cu}$ NPs in the glass composition.

The first scientific paper on metal NPs properties was written by Michael Faraday who dedicated part of his life to investigate the optical properties of metal colloids (Faraday, 1857). He was able, for example, to recognize the colors exhibited by the colloids - according to his own words - as due to "very small" (metal particles)... "as compared to the (light) wave-lengths".

In the contemporary times, metal NPs are still attracting very large interest owing to their unique physical and chemical properties. It is well known that, due to the light-metal interaction, collective oscillation of the conduction electrons, known as localized surface-plasmon (LSP), dominate the NPs optical response giving origin to the NPs colors. The LSP resonance frequency depends on the NPs compositions, as well as their shape and environment. Exploitation of LSP allows one to amplify, concentrate and manipulate light at the nanoscale, overcoming the optical diffraction limit and improving spatial resolution and sensitivity of optical probes (Novotny and Hecht, 2006). Besides the fundamental interest on the individual NPs properties, research has been dedicated also to the synthesis of new supramolecules containing metal NPs that act as optical antennas (Bharadwaj et al., 2009; Novotny and Hecht, 2006), synthesis and characterization of metaldielectric photonic nanocomposites (de Araújo and Kassab, 2016; Kassab and de Araújo, 2019), as well as fabrication of metamaterials based on metal nanostructures (Lee et al., 2014; Chen et al., 2016; Menezes et al., 2019; Yeshchenko et al., 2019). Surface-enhanced Raman spectroscopy, enhanced photoluminescence, biosensing, photovoltaic cells and thermotherapy are examples of very active areas in many laboratories (see for example: Qin and Bischof, 2012; Saha et al., 2012; Pareek et al., 2017).

In the present paper, we review some of our recent nonlinear optical experiments with colloids containing Ag nanospheres (Ag-NS) and Au nanorods (Au-NR). In one example, we demonstrated guiding and confinement of light in the core of a vortex soliton propagating in a diluted colloid containing AgNS. The other example is a detailed study of the optical nonlinearity of col- 
loids containing Au-NR. Both sets of experiments were performed using a picosecond laser operating in a single pulse regime (with low repetition rate) in order to detect only the electronic contribution to the optical response. Description of the samples preparation is given and models describing the nonlinear behavior of the samples were developed to understand the results.

\section{Materials and methods}

\section{Materials}

Chloroauric acid $\left(\mathrm{HAuCl}_{4}, 99.99 \%\right)$, silver nitrate $\left(\mathrm{AgNO}_{3}, 99.8 \%\right)$, sodium borohydride $\left(\mathrm{NaBH}_{4}, 99.99 \%\right)$, tetraoctylammonium bromide $\left(\mathrm{N}\left(\mathrm{C}_{8} \mathrm{H}_{17}\right)_{4} \mathrm{Br}\right.$, 98\%) and dodecanethiol $\left(\mathrm{CH}_{3}\left(\mathrm{CH}_{2}\right)_{10} \mathrm{CH}_{2} \mathrm{SH} \geq 98 \%\right)$ were purchased from Sigma-Aldrich. Hexadecyltrimethylammonium bromide $\left(\mathrm{C}_{16} \mathrm{TAB}\right)$, L-ascorbic acid $\left(\mathrm{C}_{6} \mathrm{H}_{8} \mathrm{O}_{6}\right)$ were purchased from Dinâmica. Deionized water (resistivity: $18 \mathrm{M} \Omega \mathrm{cm}$ ) was used in all syntheses. The magnetic stirrer and all glassware were washed with aqua-regia.

\section{Synthesis of spherical Ag nanoparticles for suspension in carbon disulfide}

Colloidal suspensions containing Ag-NS in carbon disulfide $\left(\mathrm{CS}_{2}\right)$ were prepared by a chemical synthesis method following a procedure previously reported (Reyna and de Araújo, 2014). Initially, $3.75 \mathrm{~mL}$ of a $0.03 \mathrm{~mol} / \mathrm{L}$ $\mathrm{AgNO}_{3}$ aqueous solution were added to a $0.05 \mathrm{~mol} / \mathrm{L} \mathrm{N}\left(\mathrm{C}_{8} \mathrm{H}_{17}\right)_{4} \mathrm{Br}$ solution in toluene, at a speed of $100 \mathrm{drops} / \mathrm{min}$. After $10 \mathrm{~min}, 50 \mathrm{~mL}$ of dodecanethiol (dodecanethiol-to-silver molar ratio of 2:1) was introduced in the mixture, followed by rapid addition of $3.1 \mathrm{~mL}$ of a freshly prepared $\mathrm{NaBH}_{4}$ aqueous solution $(0.4 \mathrm{~mol} / \mathrm{L})$. The reacting medium was stirred for $3 \mathrm{~h}$, and the organic layer was extracted. The resulting dodecanethiol-stabilized Ag NPs were precipitated by adding ethanol and cooling to $-18^{\circ} \mathrm{C}$ for $4 \mathrm{~h}$. Finally, the NPs were centrifuged, washed several times with ethanol, and redispersed in $\mathrm{CS}_{2}$. In order to obtain a colloidal suspension containing homogeneous distribution of Ag-NS, the product of the chemical synthesis was subjected to laser photofragmentation, under slow stirring, using the second harmonic beam at $532 \mathrm{~nm}$ from a Nd: YAG laser ( $8 \mathrm{~ns}, 85 \mathrm{~mJ} /$ pulse, $10 \mathrm{~Hz}$ ) for $1 \mathrm{~h}$. The photofragmentation of the NPs occurs due to melting and vaporization because of the large absorption of the laser energy by the NPs and low heat-transfer to the hosting medium (Takami et al., 1999; Brito-Silva et al., 2010).

\section{Synthesis of gold nanorods in aqueous solutions}

Three colloidal suspensions containing Au-NR, with different aspect ratios, were prepared by a two-step chemical synthesis: (i) the seed preparation and (ii) the nanorods growth. This seed-mediated growth method provides highyielding Au-NR with good control of size and morphology by addition of silver ions (Nikoobakht and El-Sayed, 2003). 
The first step of the synthesis consists in the preparation of gold seeds (with diameters between $2 \mathrm{~nm}$ and $4 \mathrm{~nm}$ ) from the mixture of $5 \mathrm{~mL}$ of $\mathrm{C}_{16} \mathrm{TAB}(200 \mathrm{mM})$ with $5 \mathrm{~mL}$ of $\mathrm{HAuCl}_{4}$ solution $(0.5 \mathrm{mM})$, under moderate agitation. Then, $0.600 \mathrm{~mL}$ of ice-cold $\mathrm{NaBH}_{4}(10 \mathrm{mM})$ solution was added under vigorous stirring for 2 minutes. During this time, the solution changed from colorless to brownish-yellow.

The growth solution was obtained by mixing 50,100 and $150 \mu \mathrm{L}$ of $\mathrm{AgNO}_{3}$ solution $(4 \mathrm{mM})$ in $5 \mathrm{~mL}$ of $\mathrm{C}_{16} \mathrm{TAB}(200 \mathrm{mM})$, to obtain $\mathrm{Au}-\mathrm{NR}$ with aspect ratios of 1.8, 2.8 and 3.4, respectively. Immediately $5 \mathrm{~mL}$ of $\mathrm{HAuCl}_{4}$ solution $(1 \mathrm{mM})$ followed by $70 \mu \mathrm{L}$ of L-ascorbic acid $(78.8 \mathrm{mM})$ were added to the mixture and gently stirred after each addition. With addition of $\mathrm{HAuCl}_{4}$ the solution displays an intense yellow color which changes rapidly to colorless when the L-ascorbic acid is added and after moderate shaking. Finally, $12 \mu \mathrm{l}$ of the seed solution prepared in the first step were added to the growth solution of the $\mathrm{Au}-\mathrm{NR}$, maintaining the temperature between $27^{\circ} \mathrm{C}$ and $30^{\circ} \mathrm{C}$ overnight.

All colloids were subjected to centrifugation to remove by-products and to separate the $\mathrm{Au}-\mathrm{NR}$ from spheres, cubes, prisms, and other undesirable morphologies. Three centrifugations were performed at 10,000 rpm during $30 \mathrm{~min}$, also contributing to remove the excess of $\mathrm{C}_{16} \mathrm{TAB}$. Afterwards, the supernatant was discarded and the precipitate, containing the Au-NR, was redispersed in deionized water.

\section{Morphological, quantitative and UV-VIS characterization}

The morphology and average size of the Ag-NS and Au-NR were determined by using a $120 \mathrm{kV}$ transmission electron microscope (TEM) — model: Tecnai Spirit Biotwin G2. Quantitative characterization of gold atoms contained in the Au-NR colloids was performed by using a Spectro Arcos inductively coupled plasma optical emission spectrometer (ICP-OES), with radial vision (SOP), from nanorod samples digested in aqua-regia.

The optical extinction spectra of Ag-NS and Au-NR were acquired using a ultraviolet-visible spectrophotometer (model: Varian Cary 5E) from 200 to $1500 \mathrm{~nm}$.

\section{Nonlinear optical experiments}

The experiments were performed by using the second harmonic of a Q-switched and mode-locked Nd: YAG laser ( $80 \mathrm{ps}, 532 \mathrm{~nm}$, maximum pulse energy of $10 \mathrm{~mJ}$, repetition rate: $4 \mathrm{~Hz}$ ). The closed-and open-aperture schemes of the $\mathrm{Z}$-scan technique were used to determine the nonlinear refractive indices and the nonlinear absorption coefficients, respectively (Sheik-Bahae et al., 1990; de Araújo et al., 2016). To perform these measurements, the laser beam was focused inside a $1 \mathrm{~mm}$ thick quartz cell containing the colloidal samples, by using a $5 \mathrm{~cm}$ focal length lens. Slow photodetectors placed in the far-field region with adjustable apertures in front of them were used to mea- 
sure the beam intensity transmitted by the samples. A reference channel was used to improve the signal-to-noise ratio of the measurements ( $\mathrm{Ma}$ et al., 1991; de Araújo et al., 2016).

\section{Results and discussions}

\section{Guiding and confinement of light-by-light (Reyna and de Araújo, 2016)}

Colloids containing Ag-NS suspended in acetone behave as self-defocusing media for light at $532 \mathrm{~nm}$ (Reyna and de Araújo, 2017). When the Ag-NS filling fraction is $3 \times 10^{-5}$ the colloid presents effective third-order susceptibility, $\chi_{\text {eff }}^{(3)}=-(8.3+i 2.7) \times 10^{-23} \mathrm{~m}^{2} V^{-2}$, and fifth-order effective susceptibility, $\chi_{\text {eff }}^{(5)}=+(2.8+i 0.2) \times 10^{-35} \mathrm{~m}^{4} \mathrm{~V}^{-4}$. The origin of the nonlinearity, measured using single picosecond pulses at low repetition rate, is electronic without contamination by thermal effects.

The stable propagation of vortex solitons (with unitary topological charge and $532 \mathrm{~nm}$ wavelength) was observed for $3 \mathrm{GW} / \mathrm{cm}^{2}$. The shape and radius of the vortex beam were constant for propagation over $10 \mathrm{~mm}$, which corresponds to $\sim 6$ Rayleigh lengths. The formation and propagation of the vortex soliton was corroborate by numerical simulations based on the modified nonlinear Schrödinger equation with the form $2 i k\left(\left(d E_{v}\right) /(d z)\right)+\Delta E_{v}=$ $-\left(\left(\omega^{2}\right) /\left(c^{2}\right)\right)\left[3 \chi_{\text {eff }}^{(3)} E_{v}\left|E_{v}\right|^{2}+10 \chi_{\text {eff }}^{(5)} E_{v}\left|E_{v}\right|^{4}\right]$, where $E_{v}$ is the field amplitude of the vortex beam, $\Delta$ is the Laplacian operator, $z$ is the beam propagation direction, $k=2 \pi n_{0} / \lambda, n_{0}$ is the linear refractive index, $c$ is the speed of light, $\lambda$ and $\omega$ are the laser wavelength and angular frequency, respectively. The initial condition for the vortex field was $E_{v}(r, \theta, z=0) \propto \exp \left(-\left(r^{2} / \mathrm{w}_{0}^{2}\right)+i \theta\right) \tanh$ $\left[r /\left(2 w_{v}\right)\right]$, where $r$ and $\theta$ are the polar coordinates, and $w_{0}\left(w_{v}\right)$ is the waist of the Gaussian background (vortex core).

Although the defocusing third-order contribution is dominant, the positive $\operatorname{Re}\left\{\chi_{\text {eff }}^{(5)}\right\}$ is crucial to have stable soliton propagation for long distance.

Figure 1 shows a simplified scheme of the experimental setup.

Figure 1. Simplified experimental scheme.

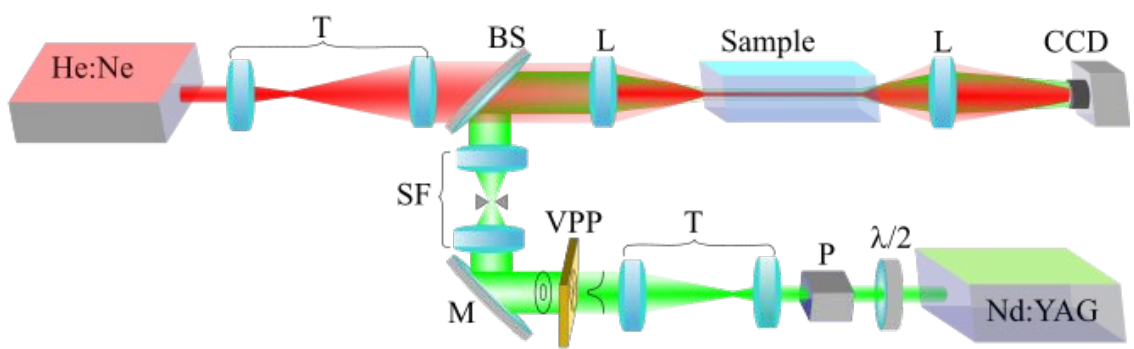

Note: P, polarizer; T, telescope; VPP, vortex phase plate; M, mirror; SF, spatial filter; BS, beam splitter and CCD (charge-coupled device) is a camera to record the transverse beam profile. The cell's length is $10 \mathrm{~mm}$. The HeNe laser has very small intensity and does not induce nonlinear effects.

Source: Creation of the authors. 
Due to the negative real part of the effective nonlinear optical susceptibility, the region illuminated by the vortex soliton has refractive index smaller than the dark core. Consequently, a weak probe beam from a $\mathrm{HeNe}$ laser was guided by the soliton as illustrated by figure 2 (a-d), which shows the probe beam profile for propagation along the colloid cell containing Ag-NS suspended in acetone. The radial intensity distribution of the weak probe beam, shown in figure $2(\mathrm{e}-\mathrm{h})$, illustrates the guiding and confinement of light induced by the optical vortex soliton.

The solid lines in figure 2 (e-h) show the intensity profiles obtained by solving two coupled nonlinear Schrödinger equations given by

$$
\begin{aligned}
2 i k_{j} \frac{\partial E_{j}}{\partial_{z}}+\Delta E_{j} & =-\frac{\omega_{j}^{2}}{c^{2}}\left\{3 \chi_{e f f}^{(3)}\left[\left|E_{j}\right|^{2}+2\left(\left|E_{G}\right|^{2} \delta_{j, v}+\left|E_{v}\right|^{2} \delta_{j, G}\right)\right]\right. \\
& \left.+10 \chi_{\text {eff }}^{(5)}\left[\left|E_{j}\right|^{4}+6\left|E_{v}\right|^{2}\left|E_{G}\right|^{2}+3\left(\left|E_{G}\right|^{4} \delta_{j, v}+\left|E_{v}\right|^{4} \delta_{j, G}\right)\right]\right\} E_{j}
\end{aligned}
$$

where $j=v, G$ correspond to the vortex soliton and the Gaussian HeNe beam, respectively. The nonlinear contribution of the Gaussian beam is negligible because $\left|E_{G}\right|^{2} \ll\left|E_{v}\right|^{2}$. The parameters used in the theoretical fit were obtained from the Z-scan experiments for $I_{v}=3 \mathrm{GW} / \mathrm{cm}^{2}$ and $I_{G}=10 \mathrm{~kW} / \mathrm{cm}^{2}$.

In summary, the propagation of the vortex soliton through the Ag-NS colloid produced a nonlinear change in the refractive index according to the vortex beam shape and an effective waveguide was formed. The imperfect coupling of the HeNe probe beam into the vortex core and the escape of light from the waveguide, due to the small refractive index difference induced be-

Figure 2. (a-d) Evolution of the transverse Gaussian ( $\mathrm{HeNe}$ ) beam profile as a function of the propagation distance $z$, when guided by the optical vortex soliton.
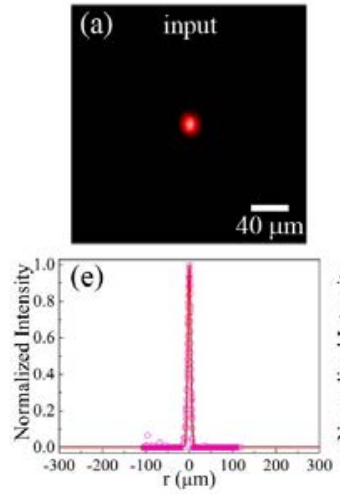$$
\text { ( }
$$
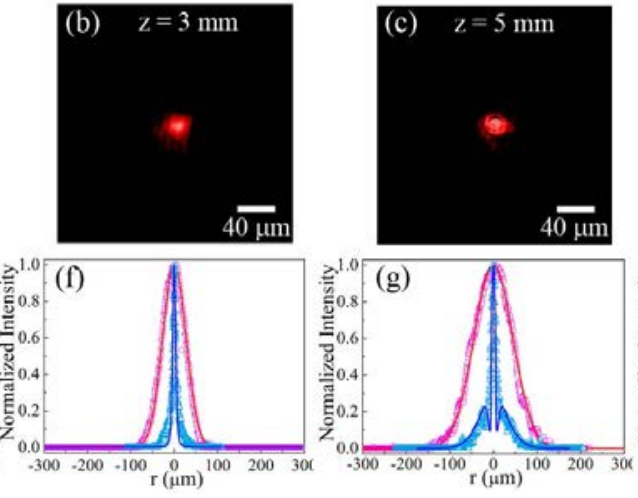

(d) $\mathrm{z}=10 \mathrm{~mm}$
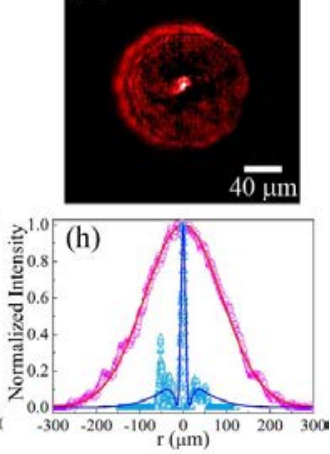

Note: (e-h) Normalized intensity profile of the Gaussian beam in absence of the vortex beam (red circles) and obtained from (a-d) (blue circles). The solid lines were obtained by numerical solutions of Eq. (1).

Source: Creation of the authors. 
tween the vortex core and the illuminated area, provided a light intensity output of $\approx 22 \%$ of the initial probe beam after $10 \mathrm{~mm}$ length propagation. By theoretical analysis of the experiments we concluded that the fifth-order refractive nonlinearity contribution is very important to obtain stable guiding and confinement of light-by-light for long distances.

The results herein reported are highly encouraging to try additional experiments in order to investigate further the light-by-light control for ultrafast signal processing using short pulse lasers propagating inside MDC.

\section{Nonlinearity management of colloidal gold nanorods (Oliveira et al., 2019)}

Several works highlight the importance of nonlinear materials based on plasmonic NPs for the development of new light-by-light control procedures due to their high optical susceptibility and ultrafast nonlinear response (Schuller et al., 2010; Lu et al., 2011; Reyna and de Araújo, 2015 and 2016). However, plasmonic NPs suffer limitation in applications that require high efficiency due to intensity losses induced by linear and nonlinear optical absorption in the NPs. In this sense, anisotropic plasmonic NPs offer significant advantages, compared with the spherical NPs, due to the ability to tune their multiple LSP resonances, enabling the study of on- and off-resonance optical phenomena. For instance, Au-NR display two tunable resonance bands associated with the electron oscillations along the transverse and longitudinal directions of the nanorods. Theoretically, by using an extension of the linear Maxwell-Garnett model, adapted for elongated metal NPs suspended in a dielectric medium, it is possible to analyze the longitudinal LSP ( $L-$ LSP) that redshift with the increase of the aspect ratio (AR) of the nanorods, as shown in figure 3 (a). The intense peak in the extinction spectrum clearly reveals that the linear optical behavior of Au-NR colloids, around the L-LSP, is dominated by absorption and/or scattering phenomena. Therefore, the AR of gold nanorods is a parameter that can be adjusted to tune the L-LSP and consequently it becomes possible to manage the intensity losses in plasmonic nanocomposites.

The LSP is also responsible for the local field enhancement, which has profound implications on the nonlinear properties of the composite medium, as can be analyzed by using the generalized Maxwell-Garnett model (Reyna and de Araújo, 2017). For example, the effective third-order susceptibility of a plasmonic colloid is given by $\chi_{e f f}^{(3)}(\omega)=\chi_{h}^{(3)}(\omega)+f \xi(\omega) \chi_{N P}^{(3)}(\omega)$, where $\chi_{h}^{(3)}(\omega)$ and $\chi_{N P}^{(3)}(\omega)$ represent the frequency dependent third-order susceptibility of the host and the nanoparticles, respectively, and $f$ is the volume fraction occupied by metal NPs. The enhancement factor, $\xi(\omega)=$ $\sum_{j, k=1}^{3}=\left[\left(\beta_{j}{ }^{2} / 3\right)\left(\left|\beta_{k}\right|^{2} / 3\right)\right]$, depends on the local field factor, $\beta_{j}(\omega)=\left(\varepsilon_{h}\right) /\left(\varepsilon_{h}\right.$ $\left.+\left(\varepsilon_{N P}-\varepsilon_{h}\right) P_{j}\right)$, which is significantly increased in the spectral region near to the LSP resonances. For metal nanorods the depolarization factors are $P_{1}=\left(\left(1-\mathrm{e}^{2}\right) / \mathrm{e}^{2}\right)[(1 / 2 \mathrm{e}) \ln ((1+\mathrm{e}) /(1-\mathrm{e}))-1]$ and $P_{2}=P_{3}=\left(\left(1-P_{1}\right) / 2\right)$ with $e=\sqrt{1-(A R)^{-2}}$, and the nonlinear behavior is enhanced in two spectral re- 
gions: near to the transverse LSP (T-LSP) and the L-LSP, while spherical NPs, with $P_{1}=P_{2}=P_{3}=1 / 3$, exhibit only one LSP resonance. Further, by writing the enhancement factor as $\xi=\sum_{j=1}^{3}\left[\left(\beta_{j}^{2} / 3\right)\left(\left|\beta_{j}^{2}\right| / 3\right)\right]+\sum_{j \neq k}\left[\left(\beta_{j}^{2} / 3\right)\left(\left|\beta_{k}\right|^{2} / 3\right)\right]$, we notice that the second term with the products $\beta_{j}\left|\beta_{k}\right|$ reveals that by varying the AR one can control the nonlinear response around both resonance regions, due to the cooperative influence of the L-LSP and T-LSP bands. Based on this fact, figure 3 (b) shows the enhancement factor dependence with the aspect ratio of Au-NR, near to the T-LSP resonance that was kept constant. Although larger values of $\xi$ are obtained when the NPs shape approaches that of a sphere ( $A R=1)$, it is possible to conclude that the AR of $\mathrm{Au}-\mathrm{NR}$ is a parameter that allows control of the nonlinearity strength, by excitation of the MDC using a laser frequency close to the T-LSP resonance.

Therefore, by exploring the region near the T-LSP band would allow to dribble the large intensity losses, due to the L-LSP band, but enjoying the effects of the nonlinearity enhancement due to the contribution of both LSP bands (Oliveira et al., 2019).

We measured the nonlinear susceptibility of aqueous colloids containing $\mathrm{Au}-\mathrm{NR}$, with three different aspect ratios, by applying the Z-scan technique, using a picosecond laser with frequency near the T-LSP resonance frequency.

Figure 4 shows the extinction spectra and TEM images of Au-NR colloids with average cross-sectional diameter of $15 \pm 0.6 \mathrm{~nm}$, corresponding to a fixed T-LSP resonance wavelength of $\approx 512 \mathrm{~nm}$, and the L-LSP resonance tuned to $610 \mathrm{~nm}, 698 \mathrm{~nm}$ and $774 \mathrm{~nm}$, by varying the aspect ratio to $1.8,2.8$ and 3.4 , respectively.

In order to compare the nonlinear response as a function of the Au-NR aspect ratio, the three colloids were diluted to exhibit the same volume fraction $\left(f=10^{-4}\right)$. Such procedure was achieved by measuring the concentration

Figure 3. Theoretical (a) linear absorption coefficient versus the wavelength and (b) enhancement factor for colloids containing gold nanorods as a function of its aspect ratio, for excitation at $532 \mathrm{~nm}$, near to the $T$-LSP resonance.

(a)

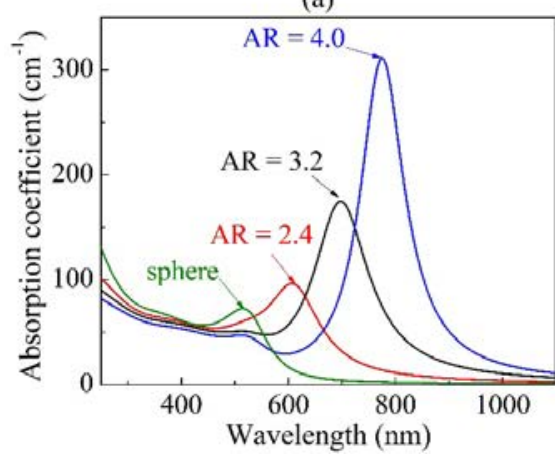

Source: Creation of the authors.

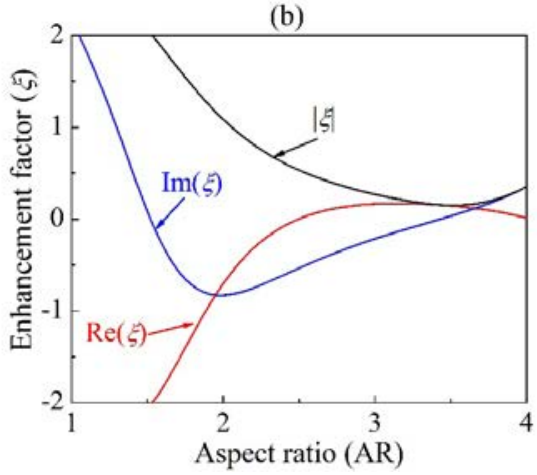


Figure 4. (a) Linear extinction spectra measured in quartz cells with thickness of $1 \mathrm{~mm}$; (b)-(d) TEM images of colloidal dispersions of gold nanorods with aspect ratios of (b) 1.8 , (c) 2.8 and (d) 3.4.
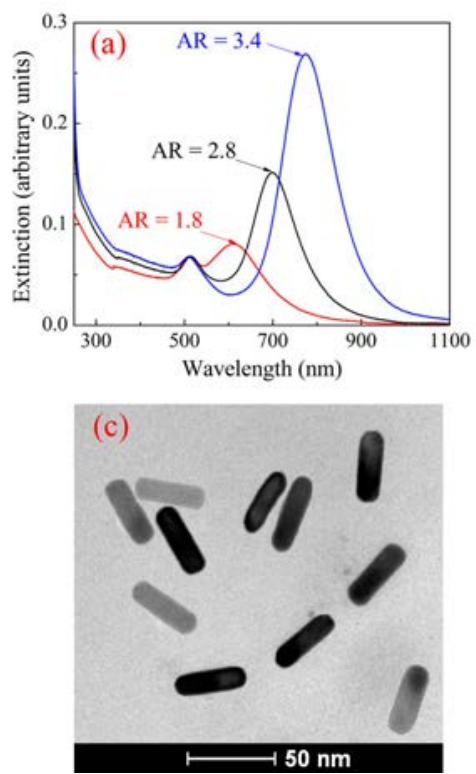

Source: Creation of the authors.
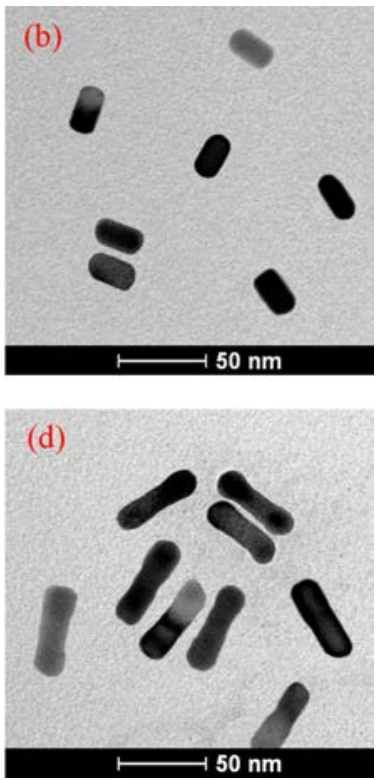

Source: Creation of the authors.

of gold atoms (12 mM), using the ICP-OES technique, and subsequently calculating the average concentration of nanorods per $\mathrm{mL}\left(1.2 \times 10^{13}\right.$ for the AR of 3.4), using the Au-NR dimensions determined from the TEM images.

Figure 5 illustrates the evolution of the transmittance profiles obtained using the open-aperture $(O A) \mathrm{Z}$-scan scheme as a function of input intensity, for Au-NR colloids. Notice that the curves show clear differences in the nonlinear behavior for different aspect ratios. For $\mathrm{AR}=3.4$ the colloid exhibits, at low intensities, a valley in the transmittance profile, related to a twophoton absorption (2PA) process. With the increase of intensity, the curves show a flat bottom valley followed by a peak formed at the bottom of the valley that indicates saturation of the 2PA process. For higher intensities, the increase in the transmittance peak indicates a saturable absorption (SA)like process related to absorption by excited-states. Colloids with Au-NR having AR equal to 2.8 and 1.8 show similar evolution up to intensities of $5.5 \mathrm{GW} / \mathrm{cm}^{2}$, but with different nonlinear coefficients (see table 1 ). The results corroborate the theoretical prediction that the nonlinear absorption, for excitation near the T-LSP band, can be controlled by changing the longitudinal dimension of the $\mathrm{Au}-\mathrm{NR}$ but keeping the rod diameter fixed.

For Au-NR with AR $=2.8$, the $Z$-scan curve in figure 5 shows a dip on the transmittance curve, at $\mathrm{z}=0$, for intensities larger than $5.5 \mathrm{GW} / \mathrm{cm}^{2}$, indicating the contribution of a new nonlinear process with origin associated to 
Figure 5. OA Z-scan profiles as a function of the input intensity, for colloids with Au-NR of different aspect ratios. The numbers above each curve represent the peak intensity in $\mathrm{GW} / \mathrm{cm}^{2}$. The curves were shifted vertically to prevent overlap between them.
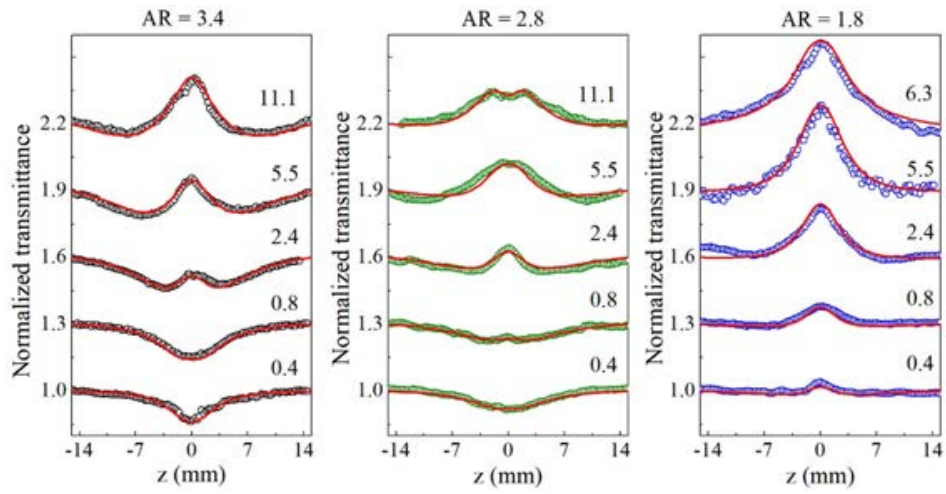

Source: Creation of the authors.

the nonlinear elastic light scattering as illustrated in figure 6. Measurements of the scattered light as a function of the input intensity were performed by placing a microscope objective and a photodetector in a direction perpendicular to the propagation of the laser beam and fixing the sample at the focus of the $5 \mathrm{~cm}$ lens, which was used for the $Z$-scan experiments.

Figure 6 shows that the variation in the AR also influences the behavior of nonlinear scattering, in the spectral region near the T-LSP resonance although the diameter of the Au-NR is not changed. The experimental results (circles), for each colloid, were fitted by linear (dashed lines), cubic (dotted lines) and quintic (solid lines) nonlinear scattering models and the best agreement was obtaining for the fifth-order process as the best fit.

From figures 5 and 6 , it was possible to adjust a mathematical expression that encompasses the nonlinear extinction exhibited by the Au-NR colloids, given by $\alpha(I)=\alpha_{0}+\alpha^{N L}(I)+\gamma_{s} I^{2}$, with $\gamma_{s}$ being the fifth-order scattering coefficient. The linear and nonlinear absorption processes are described by $\alpha_{0}$ and $\alpha^{N L}(I)=\left(\left(\alpha_{2} /\left(1+I / I_{\alpha}\right)+\left(\eta /\left(1+I / I_{\eta}\right)\right)\right.\right.$, where the first and second terms in the right hand side describe the 2PA saturation and SA-like process associated to excited-states (Cesca et al., 2012). This model was used to fit the experimental data of figure 5 using the parameters given in table 1 . The nonlinear parameters experimentally determined are very close to the theoretically estimated values for contributions of electronic transitions between the $5 \mathrm{~d}$ and $6 \mathrm{sp}$ bands (Hache et al., 1988) and hot-electron contributions, which describes the dynamics of the electronic thermalization process from the two-temperature model (Marine et al., 2013), as discussed in (Oliveira et al., 2019). Note that by controlling the AR and laser intensity (see figure 3 ) it is possible to manage the nonlinear response of the $\mathrm{Au}-\mathrm{NR}$ colloids by prevailing the SA effects (nanorods with $\mathrm{AR}=3.4$ ), the nonlinear scattering effect (nanorods with $\mathrm{AR}=2.8$, at 
Figure 6. Nonlinear scattered light intensity for the gold nanorods colloids, with different aspect ratios, versus the input intensity.

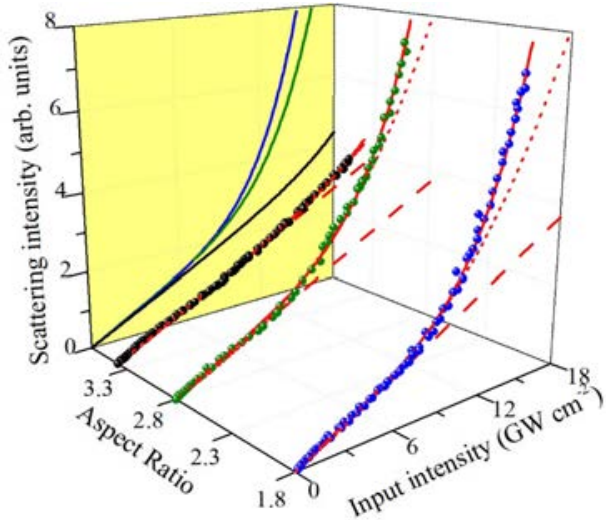

Note: The dashed, dotted and solid lines correspond to numerical fits using linear, cubic and quintic nonlinear scattering models. The lines in the yellow plane are the projections of the scattered intensities for each sample.

Source: Creation of the authors.

high intensities), or to mask the nonlinear scattering effects by the strong SA process (nanorods with $A R=1.8$ ). Based on these results, we foresee that the $\mathrm{SA}$ process in $\mathrm{Au}-\mathrm{NR}$ opens new routes to manage intensity losses effects commonly reported as one of the main limitations of metal nanoparticles in applications of light-by-light control that require high efficiency.

Figure 7 shows that colloids with Au-NR exhibit significant refractive cubic-quintic nonlinear behavior, which is revealed by the double peak-valley profile of the CA Z-scan curves as recognized also in Ag-NS experiments (Reyna and de Araújo, 2017). Hence, following the method of Gaussian decomposition and considering the simultaneous contribution of third- and fifth-order refraction, as described in Oliveira et al. (2019), it is possible to obtain the best fits for the experimental data, represented by the solid lines in figure 7. For instance, the third-order refractive indices $(5.0 \pm 0.3) \times 10^{-14}$, $(7.0 \pm 0.2) \times 10^{-14}$ and $(8.0 \pm 0.2) \times 10^{-14} \mathrm{~cm}^{2} / \mathrm{W}$ were obtained for the $\mathrm{Au}-\mathrm{NR}$ with aspect ratio of 1.8, 2.8 and 3.4, respectively, while the fifth-order re-

Table 1. Linear and nonlinear extinction coefficients for gold nanorods colloids.

\begin{tabular}{|c|c|c|c|c|c|c|}
\hline AR & $\begin{array}{c}\alpha_{0} \\
\left(\mathrm{~cm}^{-1}\right)\end{array}$ & $\alpha_{2}$ & $\left.\mathrm{w}^{-1}\right)^{\eta}$ & $\begin{array}{l}I_{\alpha} \\
\quad(G W\end{array}$ & $\mathrm{n}^{-2)}$ & $\begin{array}{c}\gamma_{s} \\
\left(\mathrm{~cm}^{3} \mathrm{GW}^{-2}\right)\end{array}$ \\
\hline 3.4 & $7.4 \pm 0.1$ & $40.0 \pm 1.3$ & $-3.0 \pm 0.1$ & $0.10 \pm 0.01$ & $3.0 \pm 0.1$ & - \\
\hline 2.8 & $8.1 \pm 0.1$ & $55.0 \pm 2.0$ & $-4.7 \pm 0.2$ & $0.04 \pm 0.01$ & $1.3 \pm 0.1$ & $0.09 \pm 0.01$ \\
\hline 1.8 & $9.2 \pm 0.1$ & $70.0 \pm 2.5$ & $-7.0 \pm 0.2$ & $0.01 \pm 0.01$ & $1.0 \pm 0.1$ & $0.12 \pm 0.02$ \\
\hline
\end{tabular}

Source: Creation of the authors. 
Figure 7. CA Z-scan curves as a function of incident laser intensities for colloids with Au-NR having different aspect ratios.
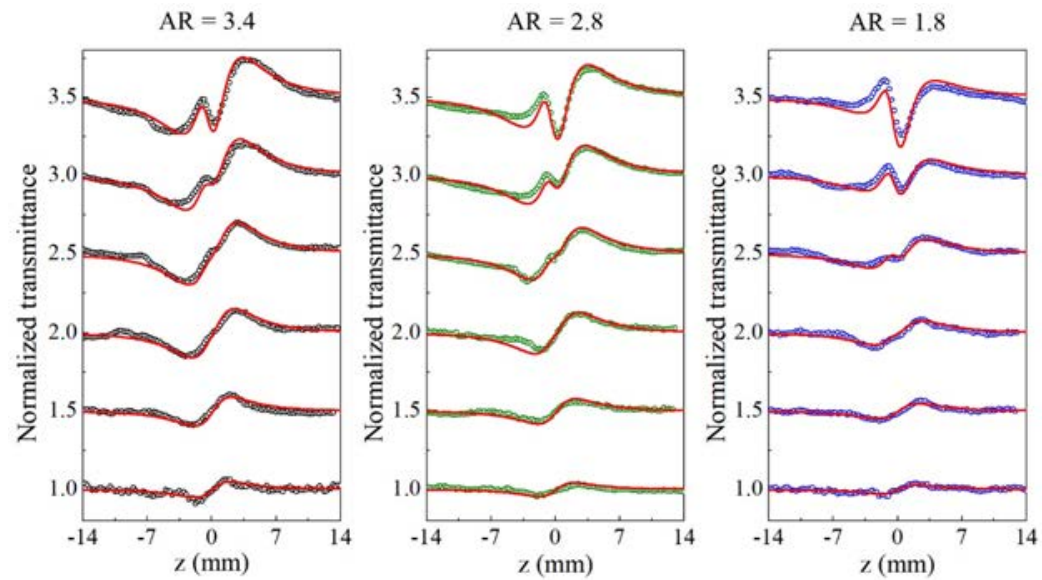

Note: From bottom to top the intensities are $0.4,0.8,1.6,2.4,3.2$ and $4 \mathrm{GW} / \mathrm{cm}^{2}$. The curves were shifted vertically for clarity.

Source: Creation of the authors.

fractive index, $(-4.6 \pm 0.4) \times 10^{-23} \mathrm{~cm}^{4} / \mathrm{W}^{2}$, remained nearly constant for all samples. The behavior of the CA Z-scan profiles reveals the strength by which high-order processes can modify the nonlinear optical behavior of Au-NR colloids.

\section{Conclusions and perspectives}

The experiments described in this short review paper illustrate how colloids with metal nanoparticles may be exploited as platforms for test of ideas related to the control of light-by-light. Besides these examples, previous publications already pointed out novel nonlinear applications and the possibility of fundamental photonic studies that can be extended to a large variety of materials containing metal nanoparticles (Garcia, 2011; Gonçalves, 2014; Reyna and de Araújo, 2017; Kassab and de Araújo, 2019). Indeed, there are many possible applications in various areas. For example, the possibility of nonlinearity management of plasmonic systems by selecting the appropriate nanoparticles concentration opens new routes for studies of high-order nonlinear optical phenomena and new spectroscopic investigations (Reyna and de Araújo, 2014; Reyna et al., 2014; Reyna and de Araújo, 2015). The combination between nonlinear optics and electronics offers the possibility for dynamical control of metal nanorods for nonlinear sensing and signaling nanotechnologies as demonstrated in (Maldonado et al., 2018 and references therein). Random lasers have been optimized by inserting metal nanoparticles in the gain media and new advances in this area are possible by 
combining metal nanorods and new optical excitation schemes (see for example: Wang et al., 2016 and 2017; Yadav et al., 2017; da Silva-Neto et al., 2019). By focusing on more fundamental aspects, we recall the large literature on multidimensional solitons and their formal analogies with ultracold bosonic gases, liquid crystals and magnetic media (Kartashov et al., 2019). Indeed, metal colloids are excellent systems for exploitation of concepts directly related to multidimensionality. Of course there are plenty of possible developments in this area as already pointed out by many authors (see for example: Walasik et al., 2017; Reyna and de Araújo, 2017; Kumar et al., 2018; Trofimov and Lysak, 2018; Kassab and de Araújo, 2019; Ortega et al., 2019, Kartashov et al., 2019, Malomed, 2019). Moreover, with new theoretical advances and the development of nanofabrication techniques exciting challenges and applications are expected for the near future.

\section{References}

Bharadwaj, P., Deutsch, B. and Novotny, L. (2009). Optical antennas. Adv. Opt. Photon., 1: 438-483. http://dx.doi.org/10.1364/AOP.1.000438

Brito-Silva, A. M., Gómez, L. A., de Araújo, C. B. and Galembeck, A. (2010). Laser ablated silver nanoparticles with nearly the same size in different carrier media. J. Nanomater. 2010: 142897. http://dx.doi.org/10.1155/2010/142897

Cesca, T., Calvelli, P., Battaglin, G., Mazzoldi, P., Mattei, G. (2012). Local-field enhancement effect on the nonlinear optical response of gold-silver nanoplanets. Opt. Express, 20: 4537-4547. http://dx.doi.org/10.1364/OE.20.004537

Chen, H.-T., Taylor, A. J. and Yu, N. (2016). A review of metasurfaces: physics and applications. Rep. Prog. Phys., 79: 076401.

http://dx.doi.org/10.1088/0034-4885/79/7/076401

Da Silva-Neto, M. L., de Oliveira, M. C. A., Dominguez, C. T., Lins, R. E. M., Rakov, N., de Araújo, C. B., Menezes, L. de S., de Oliveira, H. P. and Gomes, A. S. L. (2019). UV random laser emission from flexible $\mathrm{ZnO}$-Ag-enriched electrospun cellulose acetate fiber matrix, Sci. Reports, 9: 11765.

http://dx.doi.org/10.1038/s41598-019-48056-w

De Araújo, C. B. and Kassab, L. R. P. (2016). Enhanced photoluminescence and planar waveguide of rare earth doped germanium oxide glasses with metallic nanoparticles. In Karmakar, B., Rademann, K. and Stepanov, A. L. (eds.), Glass nanocomposites-synthesis, properties and applications. ELSEVIER.

De Araújo, C. B., Gomes, A. S. L. and Boudebs, G. (2016). Techniques for nonlinear optical characterization of materials: a review. Rep. Prog. Phys., 79: 036401. http://dx.doi.org/10.1088/0034-4885/79/3/036401

Faraday, M. (1857). Experimental relations of gold (and other metals) to light. Philos. Trans. R. Soc. 147: 145-181, London.

Garcia, M. A. (2011). Surface plasmons in metallic nanoparticles: fundamentals and applications. J. Phys. D, 44: 283001.

http://dx.doi.org/10.1088/0022-3727/44/28/283001 
Gonçalves, M. R. (2014). Plasmonic nanoparticles: fabrication, simulation and experiments, J. Phys. D, 47: 213001. https://doi.org/10.1088/0022-3727/47/21/213001

Hache, F., Ricard, D., Flytzanis, C., Kreibig, U. (1988). The optical Kerr effect in small metal particles and metal colloids: the case of gold. Appl. Phys. A, 47: 347-357. http://dx.doi.org/10.1007/BF00615498

Kartashov, Y. V., Astrakharchik, G. E., Malomed, B. A. and Torner, L. (2019). Frontiers in multidimensional self-trapping of nonlinear fields and matter. Nature Rev. Phys., 1: 185-197. http://dx.doi.org/10.1038/s42254-019-0025-7

Kassab, L. R. P. and de Araújo, C. B. (2019). Metal nanostructures for photonics. ELSEVIER.

Kumar, M., Nithyanandan, K. and Porsezian, K. (2018). Influence of spatial delay on the modulation instability in a composite system with a controllable nonlinearity. Phys. Rev. E, 97: 062208. http://dx.doi.org/10.1103/PhysRevE.97.062208

Lee, J., Tymchenko, M., Argyropoulos, C., Chen, P.-Y., Lu, F., Demmerle, F., Boehm, G., Amann, M.-C., Alù, A. and Belkin, M. A. (2014). Giant nonlinear response from plasmonic metasurfaces coupled to intersubband transitions. Nature, 511: 65-69. http://dx.doi.org/10.1038/nature13455

Lu, H., Liu, X., Wang, L., Gong, Y. and Mao, Dong. (2011). Ultrafast all-optical switching in nanoplasmonic waveguide with Kerr nonlinear resonator. Opt. Express, 19: 2910-2915. http://dx.doi.org/10.1364/OE.19.002910

Ma, H., Gomes, A. S. L. and de Araújo, C. B. (1991). Measurements of nondegenerate optical nonlinearity using a two-color single beam method. Appl. Phys. Lett., 59: 2666-2668. http://dx.doi.org/10.1063/1.105933

Malomed, B. A. (2019). Vortex solitons: old results and new perspectives. Physica D 399: 108-137. http://dx.doi.org/10.1016/j.physd.2019.04.009

Maldonado, M., Menezes, L. de S., Araujo, L. F., da Costa, G. K. B., Carvalho, I. C. S., Fontana, J., de Araújo, C. B. and Gomes, A. S. L. (2018). Nonlinear refractive index of electric field aligned gold nanorods suspended in index matching oil measured with Hartmann-Shack wavefront aberrometer, Opt. Express, 26: 20298-20305. http://dx.doi.org/10.1364/OE.26.020298

Marini, A., Conforti, M., Valle, G. D., Lee, H. W., Tran, T. X., Chang, W., Schmidt, M. A., Longhi, S., Russell, P. S. J., Biancalana, F. (2013). Ultrafast nonlinear dynamics of surface plasmon polaritons in gold nanowires due to the intrinsic nonlinearity of metals. New J. Phys., 15: No. 013033. http://dx.doi.org/10.1088/1367-2630/15/1/013033

Menezes, L. de S., Acioli, L. H., Maldonado, M., Naciri, J., Charipar, N., Rativa, D., de Araújo, C. B. and Gomes, A. S. L. (2019). Large third-order nonlinear susceptibility from a gold metasurface far off the plasmonic resonance. J. Opt. Soc. Am. B, 36: 1485-1491. http://dx.doi.org/10.1364/JOSAB.36.001485

Nikoobakht, B. and El-Sayed, M. A. (2003). Preparation and growth mechanism of gold nanorods (NRs) using seed-mediated growth method. Chem. Mater., 15: 1957-1962. http://dx.doi.org/10.1021/cm0207321 
Novotny, L. and Hecht, B. (2006). Principles of nano-optics, Cambridge Press.

Oliveira, N. T. C., Reyna, A. S., Falcão, E. H. L. and de Araújo, C. B. (2019). Light scattering, absorption, and refraction due to high-order optical nonlinearities in colloidal gold nanorods. J. Phys. Chem. C, 123: 12997-13008.

http://dx.doi.org/10.1021/acs.jpcc.9b01369

Ortega, A. B., Brambilia, E. C., Gayou, V. L., Macuil, R. D., Diaz, A. O., Alvarez, A. Z., Arzola, A. V. and Volke-Sepúlveda, K. (2019). Light control through nonlinear lensing effect in a colloid of biosynthesized gold nanoparticles, J. Modern Opt., 66: 502-511. http://dx.doi.org/10.1080/09500340.2018.1549287

Pareek, V., Bhargava, A., Gupta, R., Jain, N. and Panwar, J. (2017). Synthesis and applications of noble metal nanoparticles: a review. Adv. Sci. Eng. Med., 9: 527544. http://dx.doi.org/10.1166/asem.2017.2027

Qin, Z. and Bischof, J. C. (2012). Thermophysical and biological responses of gold nanoparticle laser heating. Chem. Soc. Rev., 41: 1191-1217. http://dx.doi.org/10.1039/c1cs15184c

Reyna, A. S. and de Araújo, C. B. (2014). Nonlinearity management of photonic composites and observation of spatial-modulation instability due to quintic nonlinearity, Phys. Rev. A, 89: 063803.

http://dx.doi.org/10.1103/PhysRevA.89.063803

Reyna, A. S., Jorge, K. C. and de Araújo, C. B. (2014). Two-dimensional solitons in a quintic-septimal medium, Phys. Rev. A, 90: 063835.

http://dx.doi.org/10.1103/PhysRevA.90.063835

Reyna, A. S. and de Araújo, C. B. (2014). Spatial phase modulation due to quintic and septic nonlinearities in metal colloids. Opt. Express, 22: 22456-22469. http://dx.doi.org/10.1364/OE.22.022456

Reyna, A. S. (2015). An optimization procedure for the design of all optical switches based on metal-dielectric nanocomposites, Opt. Express, 23: 7659-7666. http://dx.doi.org/10.1364/OE.23.007659

Reyna, A. S. (2016). Guiding and confinement of light induced by optical vortex solitons in a cubic-quintic medium. Opt. Lett., 41: 191-194. http://dx.doi.org/10.1364/OL.41.000191

Reyna, A. S. (2017). High-order optical nonlinearities in plasmonic nanocomposites-a review. Adv. Opt. Photon., 9: 720-774. http://dx.doi.org/10.1364/AOP.9.000720

Saha, K., Agasti, S. S., Kim, C., Li, X. and Rotello, V. M. (2012). Gold nanoparticles in chemical and biological sensing. Chem. Rev., 112: 2739-2779.

http://dx.doi.org/10.1021/cr2001178

Savage, G. (1975). Glass and glassware. London: Octopus Books.

Schuller, J. A., Barnard, E. S., Cai, W., Jun, Y. C., White, J. S. and Brongersma, M. L. (2010). Plasmonics for extreme light concentration and manipulation. Nature Mat., 9: 193-204. http://dx.doi.org/10.1038/nmat2630

Sheik-Bahae, M., Said, A. A., Wei, T.-H., Hagan, D. J. and Van Stryland, E. W (1990). Sensitive measurement of optical nonlinearities using a single beam. IEEE J. Quantum Electron., 26: 760-769. http://dx.doi.org/10.1109/3.53394 
Takami, A., Kurita, H. and Koda, S. (1999). Laser-induced size reduction of noble metal particles. J. Phys. Chem. B, 103: 1226-1232. http://dx.doi.org/10.1021/jp983503o

Trofimov, V. A. and Lysak, T. M. (2018). Inelastic collision of self-formed chirped solitons at a laser pulse propagation in a medium with nonlinear absorption and gold nanorods, J. Opt. Soc. Am. B, 35: 764-782. http://dx.doi.org/10.1364/JOSAB.35.000764

Walasik, W., Silahli, S. Z. and Litchinitser, N. M. (2017). Dynamics of necklace beams in nonlinear colloidal suspensions, Sci. Reports, 7: 11709. http://dx.doi.org/10.1038/s41598-017-12169-x

Wang, Z., Meng, X., Choi, S. H., Knitter, S., Kim, Y. L., Cao, H., Shalaev, V. M. and Boltasseva, A. (2016). Controlling random lasing with three-dimensional plasmonic nanorod metamaterials, Nano Lett., 16: 2471-2477. http://dx.doi.org/10.1021/acs.nanolett.6b00034

Wang, Z., Meng, X., Kildishev, A. V., Boltasseva, A. and Shalaev, V. M. (2017). Nanolasers enabled by metallic nanoparticles: from spasers to random lasers, Laser Photonics Rev., 11: 1700212. http://dx.doi.org/10.1002/lpor.201700212

Yadav, A., Zhong, L., Sun, J., Jiang, L., Cheng, G. J. and Chi, L. (2017). Tunable random lasing behavior in plasmonic nanostructures, Nano Convergence, 4: 1-8. http://dx.doi.org/10.1186/s40580-016-0095-5

Yeshchenko, O. A., Kozachenko, V. V., Tomchuk, A. V., Haftel, M., Knize, R. J. and Pinchuk, A. O. (2019). Plasmonic metasurface with tunable gap and collective surface plasmon resonance modes, J. Phys. Chem. C, 123: 13057-13062.

http://dx.doi.org/10.1021/acs.jpcc.9b02515 